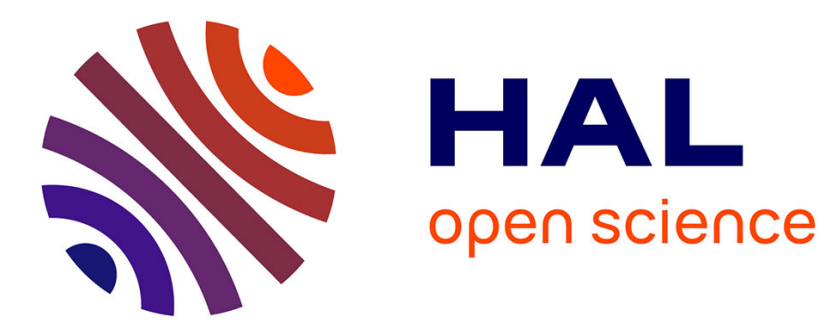

\title{
Photo-thermal Quartz Tuning Fork excitation for Dynamic Mode Atomic Force Microscope
}

Alexia Bontempi, Damien Teyssieux, Jean Friedt, Laurent Thiery, Damien Hermelin, Pascal Vairac

\section{To cite this version:}

Alexia Bontempi, Damien Teyssieux, Jean Friedt, Laurent Thiery, Damien Hermelin, et al.. Photothermal Quartz Tuning Fork excitation for Dynamic Mode Atomic Force Microscope. Applied Physics Letters, 2014, 105 (15), pp.154104. hal-02300301

\section{HAL Id: hal-02300301 https://hal.science/hal-02300301}

Submitted on 29 Sep 2019

HAL is a multi-disciplinary open access archive for the deposit and dissemination of scientific research documents, whether they are published or not. The documents may come from teaching and research institutions in France or abroad, or from public or private research centers.
L'archive ouverte pluridisciplinaire HAL, est destinée au dépôt et à la diffusion de documents scientifiques de niveau recherche, publiés ou non, émanant des établissements d'enseignement et de recherche français ou étrangers, des laboratoires publics ou privés. 


\title{
Photo-Thermal Quartz Tuning Fork excitation for Dynamic Mode Atomic Force Microscope
}

\author{
Alexia Bontempi ${ }^{1}$, Damien Teyssieux ${ }^{1}$, Jean-Michel Friedt ${ }^{2}$, Laurent Thiery ${ }^{1}$, Damien Hermelin and Pascal Vairac ${ }^{1}$ \\ ${ }^{1}$ FEMTO-ST Institute UMR 6174, Université de Franche-Comté, CNRS, \\ ENSMM, UTBM, 32 Avenue de l'Observatoire, F-25044 Besançon, France \\ ${ }^{2}$ SENSEOR SAS, Besançon, France, hosted by FEMTO-ST
}

\begin{abstract}
A Photo-thermal excitation of a Quartz Tuning Fork (QTF) for topographic studies is introduced. The non-invasive photo-thermal excitation presents practical advantages compared to QTF mechanical and electrical excitations, including the absence of the anti-resonance and its associated phase rotation. Comparison between our theoretical model and experiments validate that the optical transduction mechanism is a photo-thermal rather than photo-thermoacoustic phenomenon. Topographic maps in the context of near-field microscopy distance control have been achieved to demonstrate the performance of the system.
\end{abstract}

For nearly two decades, it has been shown that the Quartz Tuning Fork (QTF) can be used in scanning probe microscopy for the tip-sample distance control [1]. QTF is well known to have a high quality factor $Q\left(10^{5}\right.$ in vacuum and $10^{4}$ in air) and is hence considered as an alternative to the Atomic Force Microscopy (AFM) cantilever. The piezoelectric effect of the QTF allows to both excite and detect oscillations of the quartz resonator.

Several methods are employed to excite the QTF resonator. The first one is an electrical excitation, leading to AFM applications [2-5]. Thanks to the piezoelectric behavior of the substrate the QTF is made of, the setup is simplified with respect to an external optical cantilever deflection detection. However, due to the electrical capacitance which includes the contribution of the electrodes patterned along the piezoelectric substrate [6], each resonance mode is followed by an antiresonance. Despite the simple electrical implementation, several authors highlight electrical connection issues: a high parasitic capacitance (cable, electrodes, instrument) reduces the quality factor because the resonance and the antiresonance frequencies, corresponding respectively to the motional branch and the Butterworth-Van Dyke (BvD) model, become closer. Karrai and Grober [7, 8] eliminate cable capacitance by placing electronics close to the QTF. Finally, on approach to remove the antiresonance effect is by cancelling the parasitic capacitance, by adding a compensated capacitance in the electrical circuit $[3,9$ $13]$.

The mechanical excitation proposes an alternative approach to counteract these antiresonance and capacitance problems. A quartz tuning fork prong is usually glued on a supplementary actuator which vibrates at the QTF resonance frequency. This mechanical setup decreases significantly the QTF quality factor $(Q<1000)$ due to prong imbalance, yet provides a reliable tip-sample distance control mechanism in scanning probe microscopies including AFM [14-16] or Scanning Near-ficld Optical Microscopy [17].

The photo-acoustical spectroscopy (PAS) is another field in which the QTF has been successfully used. In the PAS configuration, a modulated light beam inter- acts with the QTF. It has been demonstrated recently [18] that the interaction of the QTF with the incoming light beam yielding to vibration is due to photo-thermal phenomenon inside the QTF, rather than to a photothermoacoustic one [19]. Spajer et al. show similarities between QTF voltage and stress/strain mapping, and consider the photo-thermal excitation as the main origin of the mechanical vibration.

In this letter, we illustrate this mechanical hypothesis by proposing an electrical equivalent model of the QTF photo-thermal excitation which has several advantages compared to QTF electrical and mechanical excitations. Fig. 1 shows the photo-thermal excitation setup used for topographical studies. A Quartz Tuning Fork, connected to a Lock-In Amplifier (LIA), is excited by a modulated laser beam which allows for non-invasive excitation.

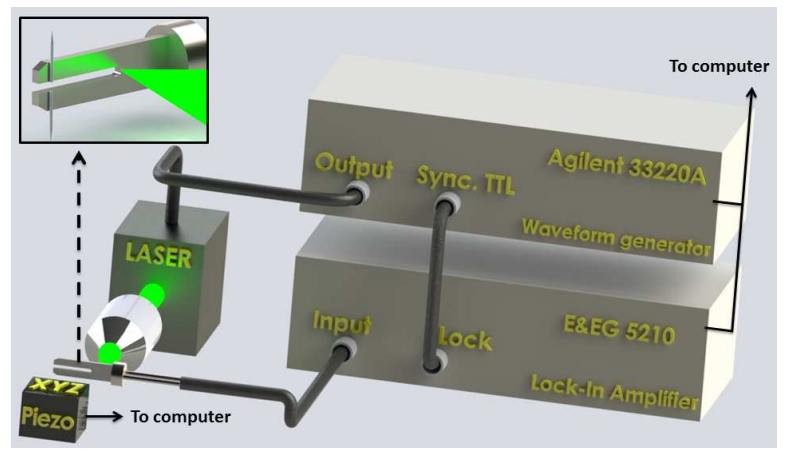

FIG. 1. Photo-thermal excitation setup

Fig. 2 presents an equivalent circuit of a tuning fork excited in a photo-thermal way and plugged in to a measuring device. It is based on the BvD circuit updated with [20] where the series $R L C$ correspond to the "motional arm" of the tuning fork. Photo-thermal excitation has already proved to be accurate for topographics studies [21]. The periodic light source is converted into local mechanical strain which implies the resonance of the structure. Quartz material being piezoelectric, voltage $\mathrm{V}$ is then extracted from the QTF. Contrary to electrical excitation, V is in series with the "motional arm". Fur- 


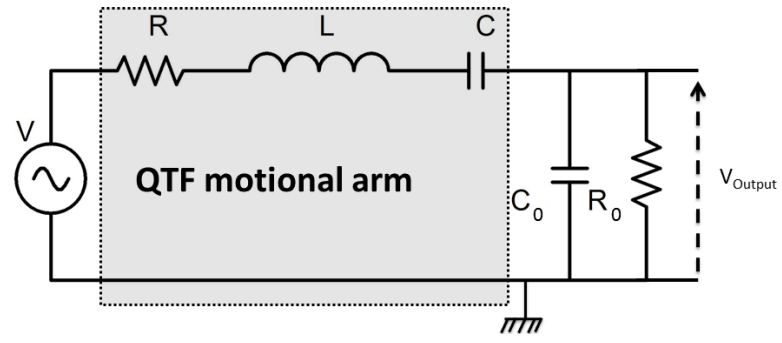

FIG. 2. Electrical equivalent circuit of the quartz tuning fork with a photo-thermal excitation

thermore, Sandoz et al. [22] show that vibration amplitude is proportional to the tuning fork voltage. $C_{0}$ is the equivalent capacitance including electrodes on the QTF for piezoelectric voltage measurement, cables and mesuring device input capacitance and $R_{0}$ is the mesuring device input impedance (LIA). The QTF transfer function is expressed in Equation 1.

$$
T=\frac{1}{\left(1+\frac{R}{R_{0}}+\frac{C_{0}}{C}-L C_{0} \omega^{2}\right)+j\left(R C_{0} \omega-\frac{1}{C R_{0} \omega}+\frac{L \omega}{R_{0}}\right)}
$$

The QTF is extracted from its metallic canister packaging, so that the quality factor drops from 100.000 to 13.000. The QTF experimental frequency response is compared in Fig. 3 with the model of Eq. 1 where the parameters have been fitted to match the experimental curve: $R=104.3 \mathrm{k} \Omega, C=3.556 \mathrm{fF}, L=6.641 \mathrm{kH}$, $C_{0}=43.154 \mathrm{pF}$ and $R_{0}=63 \mathrm{M} \Omega$. Most significantly, since the voltage source is now located in the motional branch rather than in parallel to the whole $\mathrm{BvD}$ model, we observe the absence of antiresonance which implies an unique phase rotation.

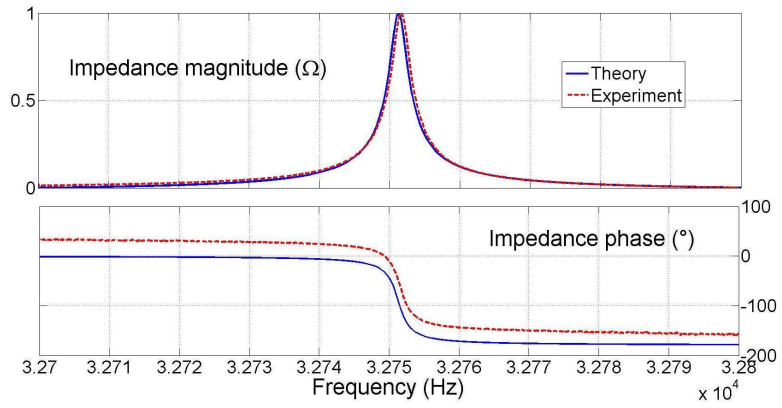

FIG. 3. QTF experimental and modeled frequency response with a photo-thermal excitation

Fig. 4 shows the theoretical QTF normalized magnitude at the resonance frequency as a function of $R_{0}$ and $C_{0}$ : the maximum value is obtained for a high resistance and a low capacitance. Fig. 5 presents the theoretical quality factor $Q$ as a function of $R_{0}$ and $C_{0}$ : for $C_{0}>1 \mathrm{nF}$, we observe an interesting area where the $Q$-factor is constant, remaining at a high value and

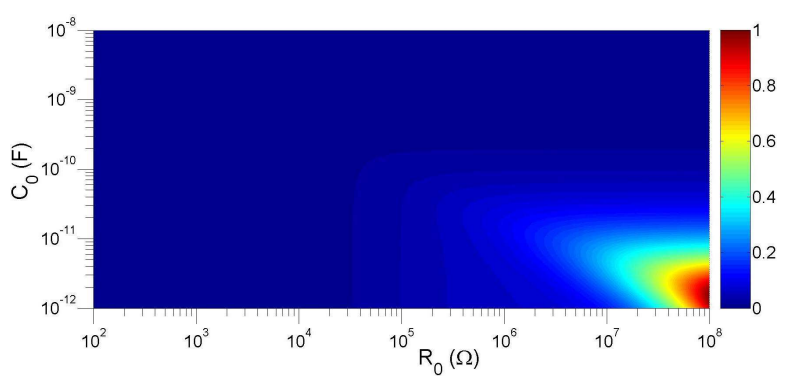

FIG. 4. Modeled normalized QTF magnitude at the resonance frequency as a function of the $C_{0}$ capacitance and $R_{0}$ resistance

hardly influenced by the instrument input impedance. For $C_{0}<1 \mathrm{nF}$, a misunderstanding on the $Q$ value can be made: $Q$ can decrease not only due to an addition of mass but also due to an electrical load problem.

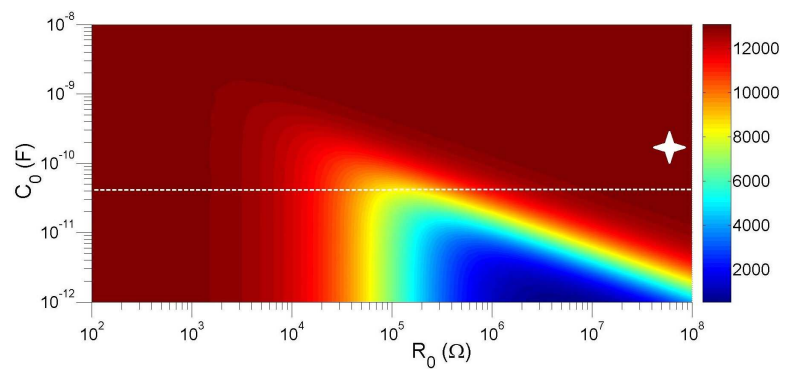

FIG. 5. Modeled $Q$ behavior as a function of the $C_{0}$ capacitance and $R_{0}$ resistance

Consequently, a tradeoff should be made between $R_{0}$ $\left(>10 \mathrm{M} \Omega\right.$ ) and $C_{0}$ to reach a good quality factor and a high QTF vibration amplitude. This point is fundamental to relate the QTF vibration magnitude with the output QTF voltage [23].

To validate the $Q$-factor model, we directly connected an unpackaged quartz tuning fork, without capacitive cable, to a lock-in amplifier (E\&EG 5210 with $40 \mathrm{pF}$ input capacitance). The total capacitance $C_{0}$, equal to $43.15 \mathrm{pF}$, is represented by the dotted line in Fig. 5. Figs. 6 and 7 show a comparison between theoretical and experimental values of the quality factor and the resonance frequency. $R_{0}$ represents the resistance added in parallel to the QTF.

The QTF electrical model with a photo-thermal excitation is in good agreement with the experimental results. This model could be adapted for a mechanical excitation as well. Consequently, a shielded cable can be used for the photo-thermal excitation which solves unwanted transfer function variabilities related to the cable and measuring instruments.

The electrical part being elucidated, we now look at the QTF mechanical part. As shown on Fig. 1, a quartz tuning fork is excited with a modulated laser beam. The QTF is considered as a coupled oscillator. It can be 


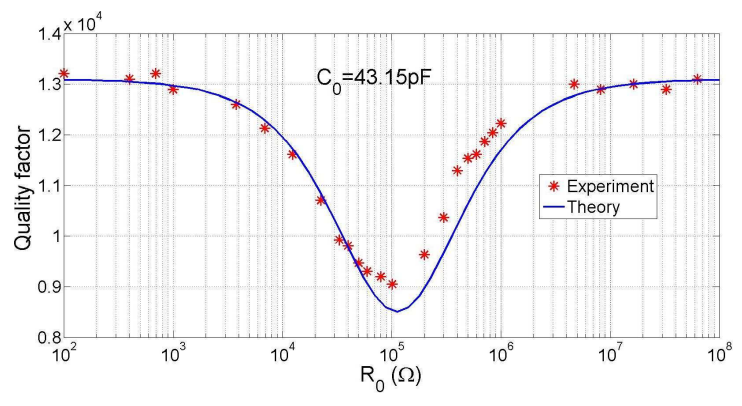

FIG. 6. $Q$ behavior as a function of $R_{0}$

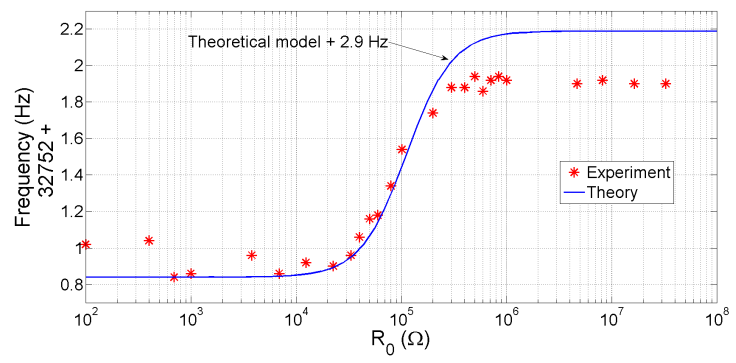

FIG. 7. Resonance frequency versus $R_{0}$

excited in two possible eigenmodes: in-phase and antiphase. In the in-phase configuration, prongs vibrate in the same direction which produces reaction forces in the QTF base. This movement causes more damping and reduces the quality factor. For the anti-phase eigenmode, prongs oscillate in a mirror pattern. The center of mass is stable, all forces are compensated inside the fork and the quality factor reaches close to 10.000 in air. By attaching a tip to one of the prongs, the symmetry is broken and the quality factor $Q$ is reduced. This phenomenon is due to the presence of reaction forces in the basis where the prongs are clamped. Rychen et al. [24] explain this $Q$ behavior by considering a third mass to their model, corresponding to the quartz tuning fork basis. To minimize the $Q$ drop, several authors $[9,24-26]$ add the same mass on the opposite free prong. The quartz tuning fork is thus balanced again and the quality factor rises.

Spajer et al. [18] provide a map where strain and stress are dominant close to the prongs basis. Based on this result, we have tested the laser beam excitation location on the QTF. We show that both quality factor and resonance frequency $f_{R}$ are slightly influenced by the location of the beam spot, particularly at the QTF prong end. We observe a $Q$ change of less than $3 \%$ and a resonance frequency shift of $1.6 \mathrm{~Hz}$ between the basis and the prong end. We studied different stages of focusing and observed no change on both $Q$ and $f_{R}$. Consequently, positioning and focusing of the laser beam slightly influence the quality factor and the resonance frequency, especially close to the QTF basis. Only the QTF voltage is mainly affected by the laser beam excitation location.

The excitation setup is also simplified and the laser will be located near the QTF basis during the following experiments. In Fig. 1, a shielded cable, connected to the QTF electrodes, is directly plugged into a lock-in amplifier. The cross in Fig. 5 corresponds to our system capacitance and LIA input impedance, respectively equal to $180 \mathrm{pF}$ and $63 \mathrm{M} \Omega$. These parameters allow for an optimal configuration in which the quality factor remains high. A laser beam is modulated at the fixed resonance frequency of the QTF, and a phase signal is extracted from the LIA. In Fig. 8 (a), a tungsten tip is glued perpendicularly on each prong to keep the quality factor as high as possible. As shown in Fig. 8 (b), the tip is sharpened with a Focus Ion Beam (FIB) method to obtain a microscopic resolution.

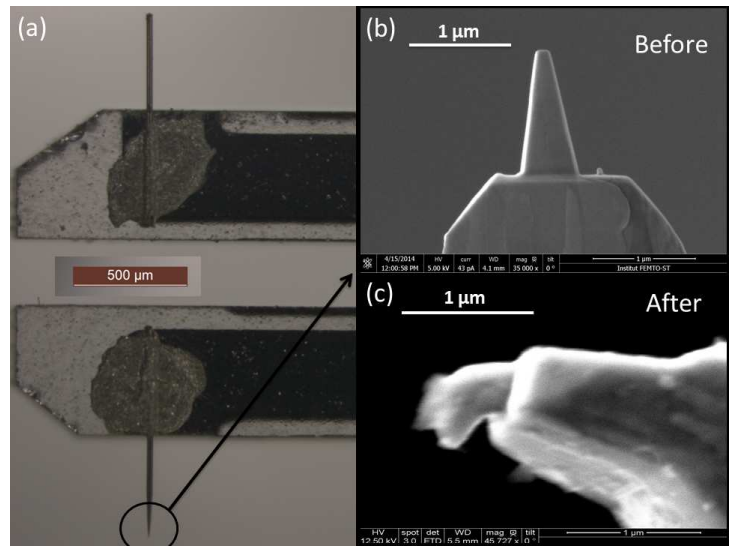

FIG. 8. (a) Tip on each prong to equilibrate the QTF, (b) Tip shape before and (c) after a scan

The laser driving signal, at the resonance frequency of the non-contact QTF, is connected to the reference input of the LIA. A sample moves toward the oscillating tip thanks to a positioner (Physik Instrumente, P-611.3S) : a phase shift is detected when the contact is established. Because of the contact, an additional force produces a resonance frequency shift. Consequently, working at fixed frequency, a phase drift is detected on the LIA. The Photo-Thermal Dynamic Mode Atomic Force Microscope (PT-DM-AFM) is also based on the phase shift with a $\Delta \Phi$ set point to control the tip to sample distance [27] and to map the surface topography.

The PT-DM-AFM system is similar to near-field imaging microscopy since the probe is a local tip that needs to be scanned over a sample.

Figure 9 shows a "T" letter topography of $600 \mathrm{~nm}$ line width. The sample is made by a $150 \mathrm{~nm}$-deep etching on $\approx 300 \mathrm{~nm}$ thick platinum layer. The quality factor of the balanced QTF is 8150 . To minimize measurement artefacts, a 4-point average filter is run by using the phong lighting texture tool of the Matlab software where the vertex normals across each face is interpolated and the reflectance at each pixel is calculated. Fig. 8 shows a comparison of the apex before 8 (b) and after 8 (c) a scan. We clearly see in the Fig. 9 the probe convolution 
due to the tip shape damaged during the scan.
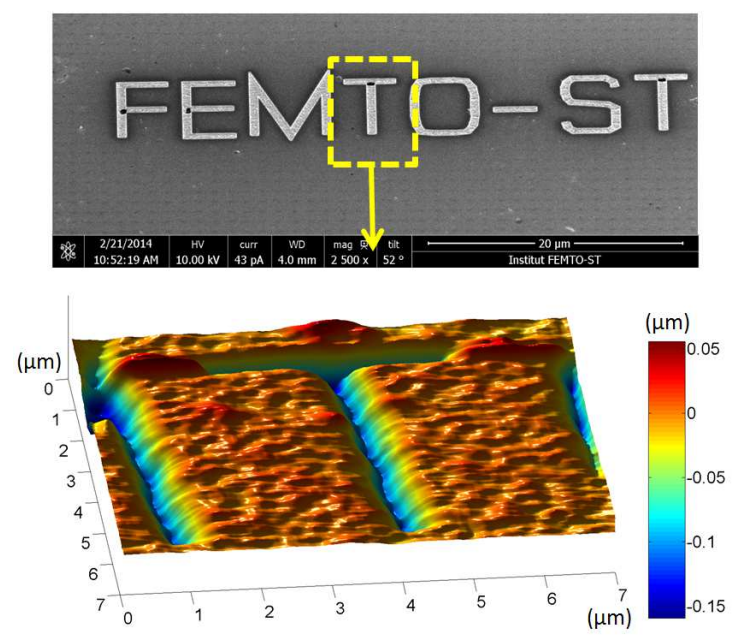

FIG. 9. T letter scanning

These results show the performance of the PT-DMAFM as topographic control system.
As a conclusion, the Photo-Thermal Dynamic Mode Atomic Force Microscope presents several advantages concerning the experimental setup. As the mechanical excitation, the absence of antiresonance allows for a tip-sampled contact detection without ambiguity on the phase. Moreover, due to the free QTF mechanical structure, a high quality factor is obtained. The system is less sensitive to electronic parameters than electrical excitation and allows for an easy identification of QTF quality factor and QTF voltage. Contrary to $[7,8]$, the electronic circuit do not need to be close to the QTF. Results of photo-thermal excitation show that laser beam positioning and focusing do not significantly affect the quality factor but only the QTF voltage. We validate our electrical model and show its reliability. Futhermore, the mechanical effect of the interaction between light and QTF has been highlighted. Finally, we present a simple and reliable excitation setup and demonstrate the performance of the PT-DM-AFM as topographic control system. We plan to use it as a force control in a scanning thermal microscope by replacing the tungsten tip with a microthermocouple [28].
[1] K. Karrai and R. D. Grober, Applied physics letters, 66, 1842 (1995).

[2] A. D. Mazzeo, A. J. Stein, D. L. Trumper, and R. J. Hocken, Precision engineering, 33, 135 (2009).

[3] T. Akiyama, N. F. de Rooij, U. Staufer, M. Detterbeck, D. Braendlin, S. Waldmeier, and M. Scheidiger, Review of Scientific Instruments, 81, 063706 (2010).

[4] D. Bayat, T. Akiyama, N. F. de Rooij, and U. Staufer, Microelectronic Engineering, 85, 1018 (2008).

[5] E. Wutscher and F. J. Giessibl, Review of Scientific Instruments, 82, 093703 (2011).

[6] J.-M. Friedt and E. Carry, American Journal of Physics, 75, 415 (2007).

[7] K. Karrai, "École Thématique du CNRS" on near-field optics, March 2000, La Londe les Maures, France (2000).

[8] R. D. Grober, J. Acimovic, J. Schuck, D. Hessman, P. J. Kindlemann, J. Hespanha, A. S. Morse, K. Karrai, I. Tiemann, and S. Manus, Review of Scientific Instruments, 71, 2776 (2000).

[9] B. P. Ng, Y. Zhang, S. Wei Kok, and Y. Chai Soh, Ultramicroscopy, 109, 291 (2009).

[10] L. González, J. Otero, G. Cabezas, and M. Puig-Vidal, Sensors and Actuators A: Physical, 184, 112 (2012).

[11] D. Stuart-Watson, A Simple Force Feedback Accelerometer Based on a Tuning Fork Displacement Sensor by in the Department of Electrical Engineering, Ph.D. thesis (2006).

[12] J. Zhao, T. Guo, L. Ma, X. Fu, and X. Hu, Sensors and Actuators A: Physical, 167, 267 (2011).

[13] Y. Qin and R. Reifenberger, Review of Scientific Instruments, 78, 063704 (2007).

[14] A. Castellanos-Gomez, N. Agrait, and G. RubioBollinger, Ultramicroscopy, 111, 186 (2011).

[15] J. Hayton, J. Polesel-Maris, R. Demadrille, M. Brun,
F. Thoyer, C. Lubin, J. Cousty, and B. Grévin, Review of Scientific Instruments, 81, 093707 (2010).

[16] F. J. Giessibl, Applied Physics Letters, 73, 3956 (1998).

[17] P. G. Gucciardi, G. Bachelier, A. Mlayah, and M. Allegrini, Review of Scientific Instruments, 76, 036105 (2005).

[18] M. Spajer, B. Cavallier, S. Euphrasie, G. Matten, X. Vacheret, P. Vairac, D. Vernier, and A. Jalocha, Applied Physics Letters, 103, 201111 (2013).

[19] C. W. Van Neste, L. R. Senesac, and T. Thundat, Applied Physics Letters, 92, 234102 (2008).

[20] S. Roundy and P. K. Wright, Smart Materials and Structures, 13, 1131 (2004).

[21] A. Labuda, K. Kobayashi, D. Kiracofe, K. Suzuki, P. Grütter, and H. Yamada, AIP ADVANCES (2011).

[22] P. Sandoz, J.-M. Friedt, and E. Carry, Review of Scientific Instruments, 79, 086102 (2008), ISSN 1089-7623.

[23] K. Karraï and R. Grober, Ultramicroscopy, 61, 197 (1995).

[24] J. Rychen, Low-Temperature Scanning Probe Microscopy and Magneto-Transport Experiments for the Local Investigation of Mesoscopic Systems Combined Abstract, Ph.D. thesis (2001).

[25] R. H. M. Smit, R. Grande, B. Lasanta, J. J. Riquelme, G. Rubio-Bollinger, and N. Agrait, Review of Scientific Instruments, 78, 113705 (2007).

[26] J. C. Acosta, G. Hwang, J. Polesel-Maris, and S. Régnier, Review of Scientific Instruments, 82, 035116 (2011).

[27] R. Garcia and A. S. Paulo, Physical Review B, 60, 4961 (1999).

[28] A. Bontempi, L. Thiery, D. Teyssieux, D. Briand, and P. Vairac, Review of Scientific Instruments, 84, 103703 (2013). 